\title{
Noncovalent interactions between cisplatin and graphene prototypes
}

\author{
Ma del Refugio Cuevas-Flores, Marco Antonio Garcia Revilla \\ Departamento de Química, Universidad de Guanajuato, Guanajuato, Mexico. \\ Massimiliano Bartolomei*, \\ Instituto de Física Fundamental, Consejo Superior de Investigaciones \\ Científicas (IFF-CSIC), Serrano 123, 28006 Madrid, Spain,
}

(Dated: May 19, 2017)

Cisplatin (CP) has been widely used as an anticancer drug for more than 30 years despite severe side effects due to its low bioavailability and poor specificity. For this reason it is paramount to study and design novel nanomaterials to be used as vectors capable to effectively deliver the drug to the biological target. The CP square-planar geometry, together with its low water solubility, suggests that it could be possibly easily adsorbed on 2D graphene nanostructures through the interaction with the related highly conjugated $\pi$-electron system. In this work pyrene has been first selected as the minimum approximation to the graphene plane, which allows to properly study the noncovalent interactions determining the $\mathrm{CP}$ adsorption. In particular, electronic structure calculations at the MP2C and DFT-SAPT levels of theory have allowed to obtain benchmark interaction energies for some limiting configurations of the $\mathrm{CP}$-pyrene complex, as well as to assess the role of the different contributions to the total interaction: it has been found that the parallel configurations of the aggregate are mainly stabilized around the minimum region by dispersion, in a similar way as for complexes bonded through $\pi-\pi$ interactions. Then, the benchmark interaction energies have been used to test corresponding estimations obtained within the less expensive DFT in order to validate an optimal exchange-correlation functional which includes corrections to take properly into account for the dispersion contribution. Reliable DFT interaction energies have been therefore obtained for CP adsorbed on graphene prototypes of increasing size, ranging from coronene, ovalene,

\footnotetext{
* Corresponding author, e-mail:maxbart@iff.csic.es
} 
and up to $\mathrm{C}_{150} \mathrm{H}_{30}$. Finally, DFT geometry optimizations and frequency calculations have also allowed a reliable estimation of the adsorption enthalpy of $\mathrm{CP}$ on graphene, which is found particularly favourable (about $-20 \mathrm{kcal} / \mathrm{mol}$ at $298 \mathrm{~K}$ and 1 bar) being double that estimated for the corresponding benzene adsorption.

PACS numbers:

KEYWORDS: cisplatin, drug delivery, graphene, two-dimensional materials, ab initio calculations, intermolecular interactions

\section{INTRODUCTION}

Graphene-based materials are strictly two-dimensional (2D) structures which represent ideal adsorbing platforms[1]: in fact, their high surface area to volume ratio, together with a wide range of facile functionalizations make them attractive as vectors for the removal and delivery of bioactive agents. Each carbon of pristine graphene is bound to three others in a $\mathrm{sp}^{2}$-hybridized flat structure offering a wide 2D area suitable for the adsorption of a large variety of molecules through non-covalent interactions, mostly determined by van der Waals forces. At present, the most common graphene-based platforms employed in bio-application are graphene oxide and reduced graphene oxide due to their favourable water solubility guaranteed by additional regions abundant in $\mathrm{sp}^{3}$-hybridized carbons with carboxyl, hydroxyl and epoxide functional groups, which moreover tend also to promote electrostatic and H-bonding interactions with the adsorbate.

As a matter of a fact, on the experimental framework it has been shown that GO platelets can act as an effective medium for the transportation of aromatic environmental pollutants[2] and elimination of organic matter[3] and antibiotics [4, 5] in water solutions. Graphene-based carriers have also emerged as optimal candidates in therapeutics delivery[6]: they have served for the transportation of water soluble[7] and insoluble[8] drugs as well as for nucleic acids loading[9].

Cis-diamminedichloro-platinum(II), or cisplatin (CP), is the most simple of the family of platinum-based anticancer drugs[10, 11] and it has been used for over 30 years as a potent 
antineoplastic agent in the treatment of a variety of tumors. In general platinum-based chemotherapy is associated with severe side effects[12] because of its poor specificity leading to systemic toxicities which badly restrict its efficacy. A possible solution for these inconvenients is the use of nanodelivery agents which are in principle able to reduce the systemic dose but increase the amount of active molecule that reaches the target site. Carbon-based nanocarriers in the form of nanotubes[13-16] and nanohorns[17, 18] have been recently experimentally proposed as host species for CP loading by exploiting the drug physical adsorption on the inner surface or outer shell of the nano-structure. In the case of graphenebased structure the CP loading has been recently attempted[19-21] mostly exploiting its covalent binding to the carbon platform. However, the low water solubility of CP together with its planar-square structure, due to the $\mathrm{dsp}^{2}$ hybridization of the central platinum atom, suggests that it could have a certain affinity with flat areas formed of $\mathrm{sp}^{2}$ carbon atoms. Therefore a favourable physical adsorption of CP on graphene-based platforms can be expected and this possibility has been not extensively addressed from the experimental point of view. Moreover, theoretical studies on the noncovalent interactions between CP and carbon nanostructures (essentially nanohorns[22] and nanotubes[16, 23]) are quite scarce and mostly rely on the DFT level of theory whose reliability, especially for the involved weak intermolecular forces, requires a proper and careful assessment. Therefore, further and more detailed studies on this topic are desirable and in this paper we aim to theoretically characterize the noncovalent interactions between $\mathrm{CP}$ and graphene prototypes. In particular, the goal is first to provide benchmark $a b$ initio calculations capable to fully assess the nature of the bonding for the physical adsorption on a small prototype; then the reference calculations also serve to validate the most suitable DFT approach which can allow to characterize the adsorption on larger and more realistic prototypes.

The $\mathrm{CP}$-pyrene complex depicted in Fig. 1, is chosen as the best prototype for carrying out benchmark quantum-chemical calculations of the involved noncovalent interactions. Pyrene $\left(\mathrm{C}_{16} \mathrm{H}_{12}\right)$ is a planar polycyclic aromatic hydrocarbon $(\mathrm{PAH})$ that can be considered as the smallest approximation of a graphene plane with the advantage that it can be treated with high level theories, which are indeed fundamental to fully recover the involved weak intermolecular interactions. For the more extended graphene prototypes, larger PAHs such as $\mathrm{C}_{24} \mathrm{H}_{12}$ (coronene), $\mathrm{C}_{32} \mathrm{H}_{14}$ (ovalene), $\mathrm{C}_{54} \mathrm{H}_{18}$ (circumcoronene), $\mathrm{C}_{96} \mathrm{H}_{24}$ (circumcircumcoronene) and $\mathrm{C}_{150} \mathrm{H}_{30}$ are considered and the main features of the related CP physical 
adsorption are also reported at the DFT level. It is found that ovalene is the most suitable $\mathrm{PAH}$ in terms of binding energy convergence and related computational cost: the most stable structure of the $\mathrm{CP}$-ovalene is determined and it is used, together with corresponding thermodynamic parameters, to obtain reliable estimations of the CP adsorption enthalpy and free energy on an extended graphene plane.

The paper is organized as follows. Section II refers the details of computational methodologies used for the electronic structure calculations. In Section III we present the results concerning first the interaction between $\mathrm{CP}$ and pyrene and then that between $\mathrm{CP}$ and larger graphene prototypes. The paper ends with Section IV, in which some conclusions are summarized.

\section{COMPUTATIONAL METHODS}

Benchmark interaction energies between CP and pyrene have been computed at the "coupled" supermolecular second-order Møller-Plesset perturbation theory (MP2C)[24] level of theory. Our choice to use the MP2C approach relies on its capability to provide accurate estimations[25] for weakly bound systems ranging from benzene-benzene[26] to rare gasgraphene prototypes[27] and molecule-graphene derivatives[28-30] at an affordable computational cost. For a proper estimation of the involved noncovalent interactions the use of large basis sets (including diffuse functions) is required. Actually, it has been shown[31] that basis sets larger than aug-cc-pVDZ[32] are needed to properly account for the interaction energy in typical noncovalently-bound complexes where dispersion plays a dominant role. We have tested that the aug-cc-pVTZ-PP[33] basis set for Pt, which includes the Stuttgart-Dresden relativistic pseudopotential[34], together with the aug-cc-pVTZ[32] set for the remaining atoms is sufficiently large to lead to well converged energies. MP2C interaction energies have been corrected for the basis set superposition error (BSSE) by the counterpoise method of Boys and Bernardi[35].

For a couple of the most attractive considered geometries additional calculations have been performed by using the density functional theory-symmetry adapted perturbation theory (DFT-SAPT)[36] approach which allows to obtain accurate results[37, 38] together with a physical decomposition of the total interaction energy in different contributions. In the DFT-SAPT method, the exchange-correlation functional has been been approximated by the 
PBE0 hybrid functional [39], whose asymptotic behavior has been corrected as described by Grüning et al[40]. In this approach, the difference between the ionization and HOMO energies of each monomer is needed; for all monomers the HOMO energies have been calculated at the DFT level of theory with the corresponding basis set as used for DFT-SAPT computations while the experimental ionization potentials for pyrene and $\mathrm{CP}$ are taken from Ref.[41] and Ref.[42], respectively.

We have gathered the components obtained from DFT-SAPT calculations in order to consider just four terms, namely, electrostatic $\left(\mathrm{E}_{\text {elst }}^{(1)}\right.$, i.e. first order electrostatic energy), exchange-repulsion ( $\mathrm{E}_{\text {exch-rep }}^{(1)}$, i.e. first order exchange-repulsion energy), induction $\left(\mathrm{E}_{i n d}^{(2)}+\mathrm{E}_{\text {exch-ind }}^{(2)}\right.$, i.e. second order induction plus exchange-induction energies), and dispersion $\left(\mathrm{E}_{\text {disp }}^{(2)}+\mathrm{E}_{\text {exch-disp }}^{(2)}\right.$, i.e. second order dispersion plus exchange-dispersion energies). Moreover, the $\delta(H F)$ contribution[43] has been also added to the DFT-SAPT energies and included in the induction term; this is a component obtained at the Hartree Fock level of theory that mostly accounts for induction and exchange-induction effects higher than second-order and which is generally small for neutral or highly symmetric systems even if not negligible.

In order to make both MP2C and DFT-SAPT calculations tractable, the density-fitting method[44] has been applied to approximate the two-electron repulsion integrals. Both MP2C and DFT-SAPT computations have been performed by using the Molpro2012.1 package[45].

Supermolecular DFT calculations of the interaction energies between CP and different PAH supports have been performed by considering the PBE[46], B3LYP[47] and M062X[48] functionals. In all cases, the reported results have been corrected for the BSSE. Moreover, PBE and B3LYP interaction energies include the latest dispersion contribution correction (D3(BJ)) of Grimme[49], which includes the damped dispersion scheme of BeckeJohnson (BJ)[50] that has demonstrated[25, 51] to lead to a better accuracy with respect to the undamped version. In the DFT computations, the Pt atom has been described by means of the Stuttgart-Dresden pseudopotential[34] whereas for the rest of the atoms the $6-311+\mathrm{G}(2 \mathrm{~d}, 2 \mathrm{p})[52]$ basis set has been used and hereafter denoted as Ia.

All reported interaction energies (except those obtained at the DFT-SAPT level) are defined as the energy difference between the complex and infinitely separated monomers having the same geometry than in the aggregate; moreover, the rigid monomers approximation is 
employed, that is all molecular internal configurations are not allowed to relax during the calculations.

In particular the following geometrical parameters have been employed: for all PAHs, the C-C bond lenghts and C-C-C angles are $1.420 \AA$ and $120^{\circ}$, respectively, whereas C-H bond lengths and C-C-H angles are $1.090 \AA$ and $120.0^{\circ}$, respectively; for $\mathrm{CP}$ the Pt-Cl, Pt-N and $\mathrm{N}-\mathrm{H}$ bond lenghts are 2.318, 2.115 and $1.010 \AA$, respectively, while the Cl-Pt-Cl, N-Pt-N, Cl-Pt-N and H-N-H angles are 95.50, 98.35, 83.08 and $108.60^{\circ}$, respectively.

In the case of the CP-ovalene system further DFT calculations by allowing the full relaxation of the monomers have been also performed: the geometry optimization together with the corresponding frequency calculations has provided the most stable cluster structure and its energy. In order to provide an estimation of the thermodynamic properties of the cluster in the gas phase at $298 \mathrm{~K}$ and 1 atm we have followed the guidelines reported in Ref.[53]: rigid rotor and harmonic oscillator approximations are assumed and frequency calculations have been performed by freezing the ovalene support while allowing the internal coordinates of the adsorbed species to relax. For the geometry optimization the 6-311+G[52] reduced basis set (denoted as $\mathbf{I b}$ ) has been employed just for atoms others than Pt, and once the stationary point has been found the corresponding energy has been evaluated with the larger Ia basis by performing single point calculations: CP adsorption enthalpy (free energy) has been determined by adding the corresponding zero-point energy and thermal (and entropy) corrections determined with the $\mathbf{I b}$ basis set to the adsorption energy $\left(\Delta \mathrm{E}_{\text {ads }}\right)$ obtained with the Ia one. $\Delta \mathrm{E}_{a d s}$ is here defined as $\mathrm{E}_{P A H-C P^{-}}\left(\mathrm{E}_{P A H^{-}} \mathrm{E}_{C P}\right)$, where $\mathrm{E}_{P A H-C P}, \mathrm{E}_{P A H}$ and $\mathrm{E}_{C P}$ are the electronic energies of the CP-PAH complex and those of the optimized isolated monomers, respectively. All DFT computations have been performed by using the Gaussian 09 code[54].

\section{RESULTS AND DISCUSSION}

\section{A. Cisplatin-pyrene interaction}

Six main configurations of the CP-pyrene complex have been chosen as the most representative to study the involved intermolecular interaction and they are shown in Fig. 1: they are two parallel (A1 and A2) and four perpendicular (B1 to B4) geometries which 
cover all possible limiting approaches of CP towards the planar support. The corresponding interaction energy profiles obtained at the MP2C level of theory are displayed in Fig. 2 as a function of the distance $R$ of the Pt atom from the center of mass of pyrene. The $R$ coordinate is varied from 8 to $2.5 \AA$ and for all configurations is set to be orthogonal to the pyrene plane. In the first panel of Fig. 2, almost equivalent energy profiles for the A1 and A2 parallel configurations can be observed: the minimum of both configurations is located at about $3.5 \AA$, but A2 displays a slightly larger interaction energy of about $10 \mathrm{meV}$. In the second panel, the profiles of the B1 and B2 perpendicular configurations are compared: the minimum of both configurations is displaced at larger distances and located around $4.75 \AA$ And it can be also observed that the B1 well is deeper than that of B2 of about $38 \mathrm{meV}$. The results for the B3 and B4 perpendicular configurations are presented in the third and fourth panels, respectively: the minimum of the energy profiles is located at even larger distances (around $5 \AA$ ) being the corresponding wells quite shallower (around -280 and $-110 \mathrm{meV}$ for B3 and B4, respectively) with respect to those for the other geometries. Globally, the A1 and A2 parallel configurations are those showing the largest interaction energies, which are around $-510 \mathrm{meV}$, while among the perpendicular ones the most attractive are the B1 and B2, that is those with the $\mathrm{NH}_{3}$ ends oriented towards the pyrene plane. Interestingly, it can be outlined that the energy difference between the most attractive parallel (A2) and perpendicular (B1) configurations is just about $24 \mathrm{meV}$, despite the related quite diverse orientations which possibly involve a different role of the competing interaction components.

In order to better assess the nature of the bonding in the A2 and B1 configurations, additional interaction energy profiles and their decomposition in physically meaningful interaction components have been obtained at the DFT-SAPT level of theory and depicted in Fig. 3. In the upper panels of Fig. 3 DFT-SAPT total interactions are compared with those obtained at the MP2C level and a good agreement can be observed along the $R$ coordinate: MP2C slightly underestimates (overestimates) the interaction obtained at the DFT-SAPT level for the A2 (B1) configuration but energy differences around the minimum are in any case about 5\%. Such results corroborate the use of the MP2C level of theory since the DFTSAPT approach, together with the aug-cc-pVTZ basis set, has proved[31] to provide reliable results whose accuracy is comparable with that of the paradigmatic CCSD(T) method.

The main four contributions (namely electrostatic, exchange repulsion, induction and dispersion) to the total interaction energy, obtained by conveniently combining (see Sec.II) 
the interaction components resulting from the DFT-SAFT partitioning scheme, are shown as function of $R$ in the lower panels of Fig. 3 and the corresponding values at selected $R$ are also reported in Table I. It can be noticed that for both configurations the main attractive contribution around the minimum comes from the dispersion component even if an important role is played by the electrostatic term, which is more important in the case of B1. Interestingly, the electrostatic contribution maintains for B1 an attractive interaction in the entire $R$ interval, becoming even more important than the dispersion one in the long range; however, for A2 it reaches slightly repulsive values for $R$ larger than about $4.5 \AA$. Such behavior can be rationalized by considering that in the case of the B1 perpendicular approach the partial positive charges of hydrogens pertaining to the $-\mathrm{NH}_{3}$ ends of cisplatin are globally closer to the $\pi$ electron system of pyrene than for A2 leading to a stronger attraction for the electrostatic contribution. The destabilizing role of the electrostatic term noticed for the A2 parallel configuration at large $R$ values is indeed analogous to that previously observed for the stacking of aromatic species such as benzene (see Fig. 8 of Ref.[55] for the benzene-benzene reference case), and it can be attributed to the long range behaviour of the quadrupole-quadrupole interaction.

The results reported in Table I for distances close to the minimum of the A2 and B1 geometries are useful to understand why similar values of the total interaction energy have been found for both configurations. Indeed, even if the sum of the exchange-repulsion and induction contributions is quite similar for both cases, the electrostatic interaction becomes more important for B1 and it partially compensates the reduction observed for the dispersion term. Moreover, although dispersion is the dominating attractive contribution for both cases, as already pointed out above, its weight is actually more important for A2, being around the minimum about $60 \%$ of the total attraction (electrostatic plus induction plus dispersion). The latter is typical of noncovalent systems bonded by $\pi-\pi$ interactions $[55,56]$ for which dispersion is by far the most important attractive component. Therefore, present analysis suggests that parallel CP-pyrene configurations mimic those for the stacking of aromatic species, such as benzene-benzene[55] and coronene-coronene [56], since a similar role of the relative interaction components is observed. 


\section{B. Cisplatin interaction with larger graphene prototypes}

Molecular prototypes larger than pyrene have to be considered in order to obtain more accurate information on the physical adsorption of cisplatin on graphene. However, if a larger approximation to the graphene plane wants to be adopted the use of less expensive electronic structure approaches is unavoidable. As a matter of fact, the results reported in the previous section confirmed the reliability of the obtained MP2C energy profiles for the CP-pyrene complex and they can be therefore safely used as reference data to validate selected DFT levels. As it is well known, standard DFT functionals such as PBE[46] and B3LYP[47] does not account for London dispersion and they are not therefore well suited to describe the noncovalent CP-pyrene interaction as shown in Fig. S1 for four selected configurations (namely, A2, B1, B3 and B4). In particular, it can be seen that the B3LYP and PBE results underestimate the reference MP2C interaction energies for all cases, while MP2 estimations, also reported, provide instead too attractive profiles: the latter is due to the well known[31] MP2 "failure", related to the overestimation of the dispersion contribution.

To possibly emend this deficiency in the PBE and B3LYP approaches we have added the corresponding D3(BJ) dispersion correction[49] and the related total interaction energies are reported in Fig. 4, together with those carried out with the M062X functional[48], which is indeed better suited to describe noncovalent aggregates since in this case the related exchange-correlation functional is fitted to reproduce dispersion near the van der Waals minimum.

It is clearly shown that the inclusion of the dispersion correction allow to obtain a better agreement with the reference MP2C profiles although it can be noticed that the B3LYPD3(BJ) approach now globally provides an overestimation, which is more significant for the A2 parallel configuration where dispersion is dominant, as seen above. The M062X estimation of the A2 reference profile is quite good in the well region while those corresponding to the perpendicular configurations (B1, B3 and B4) provide a systematic underestimation; moreover, in contrast with the dispersion corrected results, in all cases the M062X long range limit is not well described. The latter is not surprising since it is related to the notorious[31] incorrect asymptotic behavior of such reparameterized functional.

Finally, the PBE-D3(BJ) results are indeed those providing the best agreement with the $\mathrm{MP} 2 \mathrm{C}$ reference in the whole range of intermolecular distances and for all selected configura- 
tions, being the energy difference around the minimum about $2 \%$, for A2 and B1, and even less for B3 and B4. The better performance of PBE-D3(BJ) with respect to B3LYP-D3(BJ) is somewhat surprising, considering that the latter has been recently identified[25, 51] as the most favorable DFT approach in terms of the accuracy/cost ratio for the description of the intermolecular interaction in representative sets of large dispersion-stabilized noncovalent complexes. However, some of present authors have recently demonstrated[57] that the PBE-D3(BJ) level is capable to obtain a very accurate description of molecular complexes bonded by $\pi-\pi$ interactions, such as benzene-benzene and coronene-coronene, and the good agreement here obtained further support the picture emphasized above of a CP behavior similar to that of a molecule with a diffuse $\pi$ electron cloud.

From such results, it is evident that PBE-D3(BJ) is the preferred level to be used in the modeling the adsorption of cisplatin in larger graphene prototypes not only for the best accuracy along the entire energy profiles, but also for the lowest computational cost of the PBE functional, which has enabled us to consider very large carbon supports. In fact, coronene $\left(\mathrm{C}_{24} \mathrm{H}_{12}\right)$, ovalene $\left(\mathrm{C}_{32} \mathrm{H}_{14}\right)$, circumcoronene $\left(\mathrm{C}_{54} \mathrm{H}_{18}\right)$, circumcircumcoronene $\left(\mathrm{C}_{96} \mathrm{H}_{24}\right)$, and even $\mathrm{C}_{150} \mathrm{H}_{30}$ have been taken into account and for each of them the corresponding interaction with CP has been computed for a representative parallel and perpendicular configuration, as shown in Fig. 5 as a function of $R$, defined, as for the CP-pyrene case (see Figs. 2-4), as the distance between the Pt atom and the center of mass of the planar support. The considered complexes' geometries are those reported in Fig. S2 and have been chosen by selecting the most stable parallel and perpendicular approach.

In the left panel of Fig. 5 it can be seen that in the case of the parallel approach prototypes quite larger than pyrene are needed to reach a convergence in the interaction energy. As a matter of fact, even if the minimum position does not appreciably change, the corresponding interaction, also reported in Table II, becomes about $18 \%$ stronger when going from pyrene to coronene but then it tends to reach a constant value around $750 \mathrm{meV}$ for supports larger than circumcoronene.

On the contrary, it is evident that for the perpendicular approach (right panel of Fig. 5) the tendency is different: all curves, except for pyrene, are close to each other and the maximum interaction is reached for ovalene, as shown in Table II, and then gets lower going towards the larger prototypes. In any case the energy difference between ovalene and $\mathrm{C}_{150} \mathrm{H}_{30}$ around the minimum is just $15 \mathrm{meV}$ and most of the values slightly deviates around 
a central value of about $-540 \mathrm{meV}$.

This behavior can be rationalized by considering that the parallel approach is mostly dominated by dispersion, whose strength is strongly affected by the size of the $\pi$ electron system, which becomes indeed larger as the area of the prototype increases. On the other hand, the perpendicular approach is less affected by the size of carbon structure since the role of the electrostatic component is more important than for the parallel arrangement and it is expected to be depend in a different way on the area of the planar support. In fact, the electrostatic contribution is determined by the interaction between molecular permanent multipole moments whose size does not necessarily change accordingly to that of the molecules. It must be also stressed that for prototypes larger than pyrene the energy gap between the parallel and perpendicular approach significantly increases, being the former quite more stable of more than $200 \mathrm{meV}$ for circumcircumcoronene and $\mathrm{C}_{150} \mathrm{H}_{30}$.

Present analysis suggests that, in terms of the interaction energy convergence, the optimal graphene prototype would be circumcircumcoronene; however, its large size prevents the efficient computation of the complex geometry optimization, which must be accomplished to look for stationary points and to determine the related frequencies needed to obtain useful thermodynamic properties. Therefore, to this end ovalene has been instead chosen since it represents a better compromise between computational cost and accuracy. The most stable configuration of the $\mathrm{CP}$-ovalene complex, obtained starting from the parallel approach by relaxing the geometry of both cluster and monomers, has been determined and shown in Fig. 6. It can be seen that the final optimized configuration slightly deviates from the starting parallel configuration since the $-\mathrm{NH}_{3}$ ends of $\mathrm{CP}$ lean towards the ovalene plane in order to maximize the interaction between the hydrogen atoms and the $\pi$ cloud. Actually, the corresponding interaction energy, reported in Table III, is about $80 \mathrm{meV}$ deeper than that for the unoptimized parallel geometry (see Table II). Table III also summarizes the adsorption enthalpy and free energy computed at $298.15 \mathrm{~K}$ and 1 bar for the adsorption of CP on ovalene together with an estimation of the corresponding data extrapolated for the graphene plane. The latter have been obtained by scaling the $\mathrm{CP}$-ovalene results for the energy difference between the $\mathrm{CP}-$ ovalene and $\mathrm{CP}-\mathrm{C}_{150} \mathrm{H}_{30}$ interactions reported in Table II. It can be seen that the CP adsorption on graphene is significantly exothermic and exergonic, being the related adsorption enthalpy and free energy about -20 and $-10 \mathrm{kcal} / \mathrm{mol}$, respectively. These values suggest that even if the $\mathrm{CP}$ physical adsorption is mostly determined by London 
dispersion, it is particularly favourable.

In order to better assess the reliability of the provided estimations as well as to compare them with those for a typical aromatic molecule we have also obtained corresponding data for benzene, for which recent and accurate experimental values[58] related to its adsorption on graphene are available. To do that, an optimization of the benzene-ovalene complex has been achieved (see Fig. S3) and the related thermodynamic properties are shown in Table III together with the extrapolated values for the adsorption on the graphene plane (see also Supplementary Material). It can be seen that the adsorption enthalpy for benzene-graphene is about $1.8 \mathrm{kcal} /$ mole less negative than the experimental findings[58] and this discrepancy can be explained as follows. On the one hand, the harmonic oscillator approximation here considered can lead to an underestimation of the calculated enthalpy and free energy; on the other hand, and more importantly, a real graphene monolayer is actually not perfectly planar since it tends to form wrinkles[59] loosely distributed on the basal plane and leading to groove regions which are regarded as high affinity adsorption sites[60]. In fact, if the species is adsorbed on a groove region the resulting interaction can be quite enhanced with respect to a perfectly flat surface. In order to demonstrate this effect, further calculations for the adsorption of benzene on a concave side of a curved 3D ovalene structure have been performed: we have found that the related interaction energy can be up to $2.0 \mathrm{kcal} / \mathrm{mole}$ more attractive than that for the planar ovalene. These findings lead to a probable explanation for the gap found with the experimental data of Ref.[58] and also to a validation of present method to obtain thermodynamic properties for the physical adsorption of CP and benzene on graphene structures. In fact, although it is clear that present estimations lead to a lower limit of the (absolute) adsorption energy for a real (wrinkled) graphene monolayer, we can safely conclude that CP physisorption must be very favourable being the related enthalpy and free energy variation more than double that for benzene, as seen in Table III.

\section{CONCLUSIONS}

Benchmark first principles energies have been obtained for the interaction between CP and pyrene, considered a minimum graphene prototype. The obtained energy profiles, calculated for different limiting configurations of the CP-pyrene complex, have served to assess the nature of the involved noncovalent bonding: parallel geometries are those showing the largest 
attraction and they are mostly stabilized by dispersion in a similar manner as for complex bonded through $\pi-\pi$ interactions. The PBE-D3(BJ) level of theory has been then validated as that providing the better agreement with the benchmark interaction energies and it has subsequently allowed the study of the interaction of CP with larger graphene prototypes up to $\mathrm{C}_{150} \mathrm{H}_{30}$. Ovalene has been found as the prototype exhibiting the best compromise between computational cost and accuracy and has permitted a reliable estimation of the adsorption enthalpy (at standard temperature and pressure) of CP on graphene. The reported value is very favourable (about $-20 \mathrm{kcal} / \mathrm{mol}$ ), doubling that estimated for an archetypical aromatic species such as benzene, and we can foresee to be comparable with that for the adsorption of small PAHs such as naphtalene, anthracene and pyrene. Present findings demonstrate that CP can be easily physically adsorbed on graphene and graphene-derived flakes having large $\mathrm{sp}^{2}$ areas on the basal plane. The propensity for the CP adsorption on graphene-oxide prototypes as well as that in a water solution medium is clearly worth to be investigated and work in these directions is in progress.

\section{Acknowledgments}

The work has been funded by the Spanish grant FIS2013-48275-C2-1-P. Allocation of computing time by CESGA (Spain) is also acknowledged. M.R.C-F. acknowledges financial support from the i-COOP +2015 program of Consejo Superior de Investigaciones Científicas, CONACYT (241710), University of Guanajuato (DAIP, NUA 136722) and University of Zacatecas.

\section{Supplementary Material}

Additional figures showing further DFT and MP2 interaction energies for the CP-pyrene complex, the investigated CP-PAHs configurations, and the benzene-ovalene most stable structure. Additional Table with interaction energies for the benzene-ovalene and benzene$\mathrm{C}_{150} \mathrm{H}_{30}$ complexes. This information is available free of charge via the Internet.

[1] R. Balasubramanian and S. Chowdhury, J. Mater. Chem. A 3, 21968 (2015). 
[2] T. Lammel, P. Boisseaux, and J. M. Navas, Environ. Toxicol. 30, 1192 (2014).

[3] H. Ren, D. D. Kulkarni, R. Kodiyath, W. Xu, I. Choi, and V. V. Tsukruk, ACS Appl. Mater. Interfaces 6, 2459 (2014).

[4] Y. Gao, Y. Li, L. Zhang, H. Huang, J. Hu, S. M. Shah, and X. Su, J. Colloid Interface Sci. 368, 540 (2012).

[5] X. Zhang, J. Shen, N. Zhuo, Z. Tian, P. Xu, Z. Yang, and W. Yang, ACS Appl. Mater. Interfaces 8, 24273 (2016).

[6] C. McCallion, J. Burthem, K. Rees-Unwin, A. Golovanov, and A. Pluen, Eur. J. Pharm. Biopharm. 104, 235 (2016).

[7] X. Yang, X. Zhang, Z. Liu, Y. Ma, Y. Huang, and Y. Chen, J. Phys. Chem. C 112, 17554 (2008).

[8] Z. Liu, J. T. Robinson, X. Sun, and H. Dai, J. Am. Chem. Soc. 130, 10876 (2008).

[9] Z. Liu, L. Zhao, Z. Zhou, T. Sun, and Y. Zu, Scanning 34, 302 (2012).

[10] X. Wang and Z. Guo, Chem. Soc. Rev. 42, 202 (2013).

[11] T. C. Johnstone, K. Suntharalingam, and S. J. Lippard, Chem. Rev. 116, 3436 (2016).

[12] C. A. Rabik and M. E. Dolan, Cancer Treat. Rev. 33, 9 (2007).

[13] C. Tripisciano, S. Costa, R. J. Kalenczuk, and E. Borowiak-Palen, Eur. Phys. J. B 75, 141 (2010).

[14] A. Guven and L. J. W. I. A. Rusakova, M. T. Lewis, Biomaterials 33, 1455 (2012).

[15] A. Kazemi-Beydokhti, S. Z. Heris, and M. R. Jaafari, Chem. Eng. Res. Des. 112, 56 (2016).

[16] L. A. D. Souza, C. A. S. Nogueira, P. F. R. Ortega, J. F. Lopes, H. D. Calado, R. L. Lavall, G. G. Silva, H. F. D. Santos, and W. B. D. Almeida, Inorg. Chim. Acta 447, 38 (2016).

[17] K. Ajima, M. Yudasaka, T. Murakami, A. Maigné, K. Shiba, and S. Iijima, Mol. Pharm. 2, 475 (2005).

[18] K. Ajima, T. Murakami, Y. Mizoguchi, K. Tsuchida, T. Ichihashi, S. Iijima, and M. Yudasaka, ACS Nano 2, 2057 (2008).

[19] L. Tian, X. Pei, Y. Zeng, R. He, Z. Li, J. Wang, Q. Wan, and X. Li, J. Nanopart. Res. 16, 2709 (2014).

[20] C. C. Ciobotaru, C. M. Damian, E. Matei, and H. Iovu, Mat. Plast. 51, 75 (2014).

[21] G. Y. Chen, C. L. Meng, K. C. Lin, H. Y. Tuan, H. J. Yang, C. L. Chen, K. C. Li, C. S. Chiang, and Y. C. Hu, Biomaterials 40, 12 (2015). 
[22] L. A. D. Souza, C. A. S. Nogueira, J. F. Lopes, H. F. D. Santos, and W. B. D. Almeida, J. Inorg. Biochem. 129, 71 (2013).

[23] Z. Mahdavifar and R. Moridzadeh, J. Incl. Phenom. Macrocycl. Chem. 79, 443 (2014).

[24] M. Pitonák and A. Hesselmann, J. Chem. Theory Comput. 6, 168 (2010).

[25] R. Sedlak, T. Janowski, M. Pitonak, J. Rezac, P. Pulay, and P. Hobza, J. Chem. Theory Comput. 9, 3364 (2013).

[26] M. Bartolomei, F. Pirani, and J. M. C. Marques, J. Comput. Chem. 36, 2291 (2015).

[27] M. Bartolomei, E. Carmona-Novillo, M. I. Hernández, J. Campos-Martínez, and F. Pirani, J. Phys. Chem. C 117, 10512 (2013).

[28] M. Bartolomei, E. Carmona-Novillo, M. I. Hernández, J. Campos-Martínez, F. Pirani, G. Giorgi, and K. Yamashita, J. Phys. Chem. Lett. 5, 751 (2014).

[29] M. Bartolomei, E. Carmona-Novillo, and G. Giorgi, Carbon 95, 1076 (2015).

[30] M. Bartolomei and G. Giorgi, ACS Appl. Mater. Interfaces 8, 27996 (2016).

[31] K. E. Riley, M. Pitonak, P. Jurecka, and P. Hobza, Chem. Rev. 110, 5023 (2010).

[32] R. A. Kendall, T. H. Dunning, and R. J. Harrison, J. Chem. Phys. 96, 6796 (1992).

[33] D. Figgen, K. A. Peterson, M. Dolg, and H. Stoll, J. Chem. Phys. 130, 164108 (2009).

[34] D. Andrae, U. Haeussermann, M. Dolg, H. Stoll, and H. Preuss, Theor. Chim. Acta 77, 123 (1990).

[35] S. Boys and F. Bernardi, Mol. Phys. 19, 553 (1970).

[36] A. Hesselmann, G. Jansen, and M. Schütz, J. Chem. Phys. 122, 014103 (2005).

[37] A. Hesselmann and T. Korona, Phys. Chem. Chem. Phys. 13, 732 (2011).

[38] A. Hesselmann and T. Korona, J. Chem. Phys. 141, 094107 (2014).

[39] C. Adamo and V. Barone, J. Chem. Phys. 110, 6158 (1999).

[40] M. Grüning, O. Gritsenko, S. van Gisergen, and E. Baerends, J. Chem. Phys. 114, 652 (2001).

[41] NIST Chemistry WebBook, NIST Standard Reference Database Number 69, ed. P. J. Linstrom and W. G. Mallard, 2007, http://WebBook.nist.gov.

[42] B. ywicka and P. Moejko, Eur. Phys. J. D 66, 54 (2012).

[43] R. Moszynski, T. Heijmen, and B. Jeziorski, Mol. Phys. 88, 741 (1996).

[44] O. Vahtras and J. A. an M.W. Feyereisen, Chem. Phys. Lett. 213, 514 (1993).

[45] H.-J. Werner, P. J. Knowles, R. Lindh, F. R. Manby, M. Schütz, P. Celani, T. Korona, G. Rauhut, R. D. Amos, A. Bernhardsson, et al., Molpro, version2012.1, a package of ab 
initio programs (2012), seehttp://www.molpro.net.

[46] J. Perdew, K. Burke, and M. Ernzerhof, Phys. Rev. Lett. 77, 3865 (1996).

[47] P. J. Stephens, F. J. Devlin, C. F. Chabalowski, and M. J. Frisch, J. Phys. Chem. 98, 11623 (1994).

[48] Y. Y. Zhao and D. G. Truhlar, Theoretical Chemistry Accounts 120, 215 (2008).

[49] S. Grimme, S. Ehrlich, and L. Goerigk, J. Comput. Chem. 32, 1456 (2011).

[50] E. R. Johnson and A. D. Becke, J. Chem. Phys. 124, 174104 (2006).

[51] L. Goerigk, H. Kruse, and S. Grimme, ChemPhysChem 12, 3421 (2011).

[52] J. S. Binkley, J. A. Pople, and W. J. Hehre, J. Am. Chem. Soc. 102, 939 (1980).

[53] P. Lazar, F. Karlicky, P. Jurecka, M. Kocman, E. Otyepkova, K. Safarova, and M. Otyepka, J. Am. Chem. Soc. 135, 6372 (2013).

[54] M. J. Frisch, G. W. Trucks, H. B. Schlegel, G. E. Scuseria, M. A. Robb, J. R. Cheeseman, G. Scalmani, V. Barone, B. Mennucci, G. A. Petersson, et al., Gaussian 09 Revision E.01, gaussian Inc. Wallingford CT 2009.

[55] M. O. Sinnokrot and C. D. Sherrill, J. Phys. Chem. A 108, 10200 (2004).

[56] R. Podeszwa, J. Chem. Phys. 132, 044704 (2010).

[57] M. Bartolomei, F. Pirani, and J. Marques, J. Phys. Chem. C pp. xx-xx (2017).

[58] E. Otyepkova, P. Lazar, K. Cepe, O.Tomanec, and M. Otyepka, Applied Materials Today 5, $142(2016)$.

[59] J. C. Meyer, A. K. Geim, M. I. Katsnelson, K. S. Novoselov, T. J. Booth, and S. Roth, Nature 446, 60 (2007).

[60] J. Wang, Z. Chen, and B. Chen., Energy Environ. Sci. 48, 4817 (2014). 
TABLE I: DFT-SAPT contributions to the total interaction energies for the A2 and B1 CP-pyrene geometries (see Fig. 1). The reported values refers to intermolecular distances equals to 3.5 and $4.5 \AA$, roughly corresponding to the equilibrium distances at DFT-SAPT level for the A2 and B1 configurations (see Fig. 3), respectively. Induction and dispersion components also include the respective exchange contributions and the $\delta(\mathrm{HF})$ term is also added to the induction contribution (see Section II). All values are in meV.

\begin{tabular}{ccc}
\hline & $\mathrm{A} 2(3.5 \AA)$ & $\mathrm{B} 1(4.5 \AA)$ \\
\hline electrostatic & -279.6 & -388.0 \\
exchange repulsion & 672.8 & 611.9 \\
induction & -214.9 & -170.6 \\
dispersion & -713.6 & -530.3 \\
\hline Total & -535.3 & -477.0 \\
\hline
\end{tabular}

TABLE II: Total energy for the interaction between CP and graphene molecular prototype of increasing size, namely pyrene $\left(\mathrm{C}_{16} \mathrm{H}_{10}\right)$, coronene $\left(\mathrm{C}_{24} \mathrm{H}_{12}\right)$, ovalene $\left(\mathrm{C}_{32} \mathrm{H}_{14}\right)$, circumcoronene $\left(\mathrm{C}_{54} \mathrm{H}_{18}\right)$, circumcircumcoronene $\left(\mathrm{C}_{96} \mathrm{H}_{24}\right)$ and $\left.\mathrm{C}_{150} \mathrm{H}_{30}\right)$ computed at the PBE-D3(BJ) level of theory for the most favourable parallel and perpendicular approach (see Fig. S2). The reported values refer to intermolecular distances equals to 3.5 and $4.75 \AA$, roughly corresponding to the equilibrium distances at the PBE-D3(BJ) level for the parallel and perpendicular configurations(see Fig. 5), respectively. All values are in meV and interaction energies uncorrected for the BSSE are also given in parentheses.

\begin{tabular}{|c|c|c|c|}
\hline & \multicolumn{3}{|c|}{ parallel $(3.5 \AA)$ perpendicular $(4.75 \AA)$} \\
\hline $\mathrm{C}_{16} \mathrm{H}_{10}$ & -522.2 & -503.5 & \\
\hline $\mathrm{C}_{24} \mathrm{H}_{12}$ & -618.0 & -523.9 & \\
\hline $\mathrm{C}_{32} \mathrm{H}_{14}$ & $-664.9(-778.9)$ & -540.4 & $(-596.8)$ \\
\hline $\mathrm{C}_{54} \mathrm{H}_{18}$ & -716.0 & -534.8 & \\
\hline $\mathrm{C}_{96} \mathrm{H}_{24}$ & -742.1 & -530.8 & \\
\hline $\mathrm{C}_{150} \mathrm{H}_{30}$ & $-749.0(-883.4)$ & -525.2 & $(-588.1)$ \\
\hline
\end{tabular}


TABLE III: Interaction energy $\left(\mathrm{E}_{\text {int }}\right)$ and adsorption energy $\left(\Delta \mathrm{E}_{a d s}\right)$, enthalpy $\left(\Delta \mathrm{H}_{a d s}\right)$, and free energy $\left(\Delta \mathrm{G}_{a d s}\right)$ for the sorption of CP on ovalene and graphene supports. Related values for benzene are also reported for comparison. $\Delta \mathrm{H}_{a d s}$ and $\Delta \mathrm{G}_{a d s}$ refers to $298.15 \mathrm{~K}$ and 1 bar and have been obtained by assuming rigid rotor and harmonic frequency approximations through PBE-D3(BJ) calculations by considering the carbon support as a rigid structure. Values for the adsorption on graphene are obtained by scaling the adsorption energies by using the interaction energies computed for ovalene and $\mathrm{C}_{150} \mathrm{H}_{30}$ (see text and Table II). All values are in meV.

\begin{tabular}{lrrrl}
\hline & $\mathrm{E}_{\text {int }}$ & $\Delta \mathrm{E}_{a d s}$ & $\Delta \mathrm{H}_{a d s}$ & \multicolumn{1}{c}{$\Delta \mathrm{G}_{a d s}$} \\
\hline CP-ovalene & $-744.7-839.3$ & -785.8 & -324.7 \\
CP-graphene & -943.3 & -889.7 & -428.8 \\
benzene-ovalene & -381.2 & -424.3 & -376.0 & -67.4 \\
benzene-graphene & -487.2 & $-438.9(-516 \pm 13)^{a}$ & $-130.3(-154 \pm 13)^{a}$ \\
\hline
\end{tabular}

${ }^{a} \overline{\text { Experimental values from Ref.[58] obtained for T between } 313 \text { and } 363} \mathrm{~K}$. 

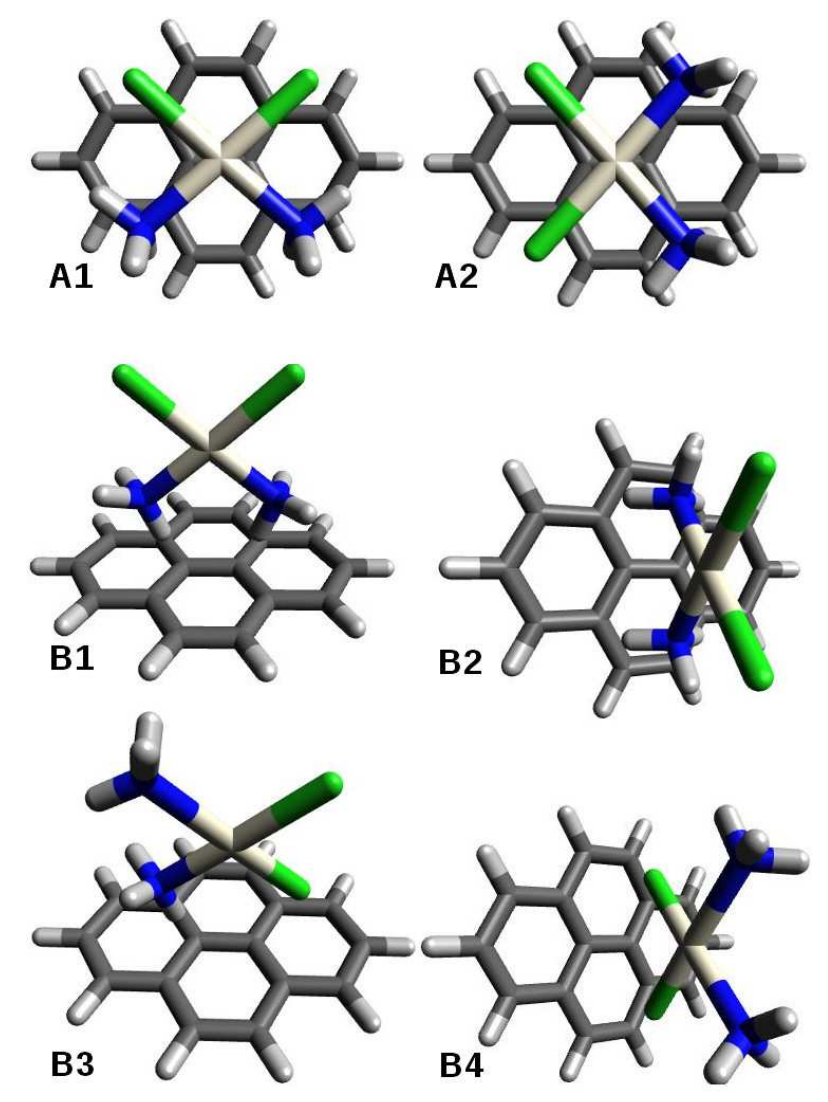

FIG. 1: Selected limiting configurations for the CP-pyrene complex. The Pt atom is at the center of the planar-square structure of $\mathrm{CP}$, whose $\mathrm{Cl}$ and $\mathrm{N}$ atoms are represented as green and blue sticks, respectively. A1 and A2 represent two different parallel configurations. Those from B1 to B4 correspond to four different perpendicular geometries: in the case of $\mathrm{B} 1$ and $\mathrm{B} 2$ the $-\mathrm{NH}_{3}$ ends of $\mathrm{CP}$ are oriented towards the pyrene plane; for $\mathrm{B} 3$ one $-\mathrm{NH}_{3}$ and one $-\mathrm{Cl}$ end are turned towards the plane while for $\mathrm{B} 4$ both $-\mathrm{Cl}$ ends are oriented downwards. 


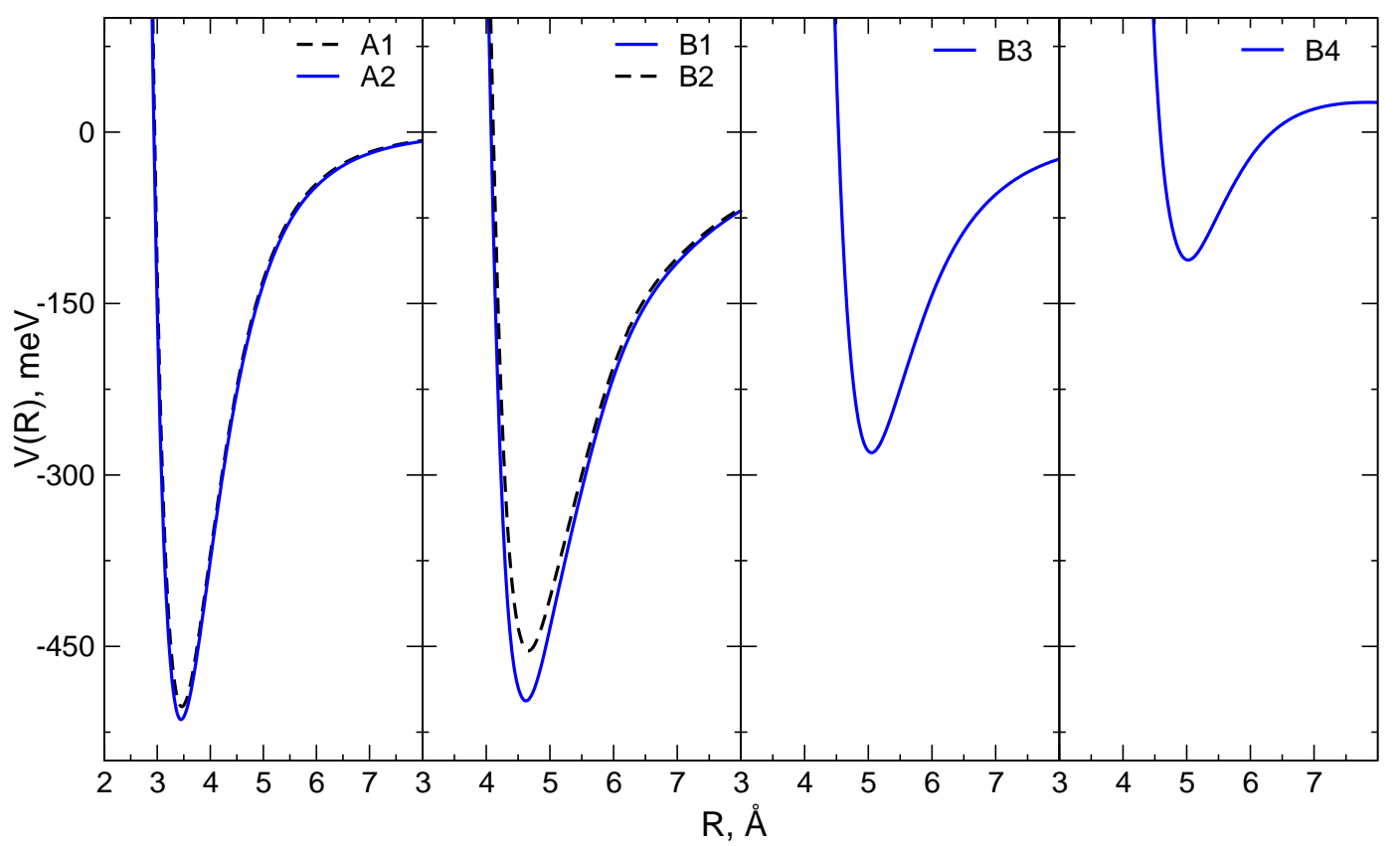

FIG. 2: Interaction energies as a function of the intermolecular distance $R$ obtained at the MP2C level for the different parallel and perpendicular configurations of the CP-pyrene complex shown in Fig.1. 


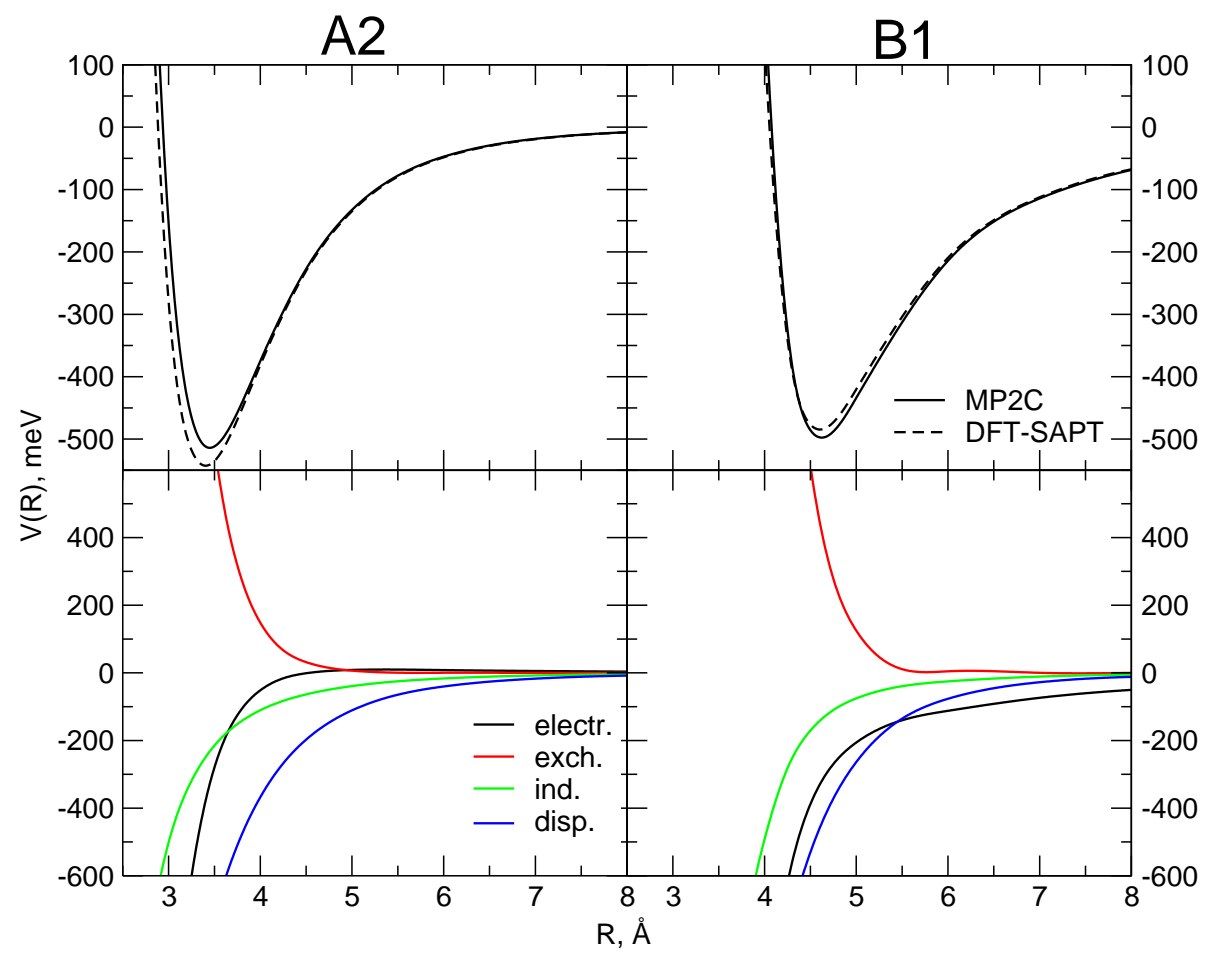

FIG. 3: Upper panels: comparison between MP2C and DFT-SAPT interaction energies for the A2 and B1 configurations (see Fig.1) of the CP-pyrene complex as a function of the intermolecular distance $R$. Lower panels: corresponding DFT-SAPT contributions (electrostatic, exchange repulsion, induction, dispersion) to the total interaction energy as a function of the intermolecular distance. The induction and dispersion profiles also include the respective exchange contributions. and the $\delta(\mathrm{HF})$ term is also included in the induction contribution(see Section II). 


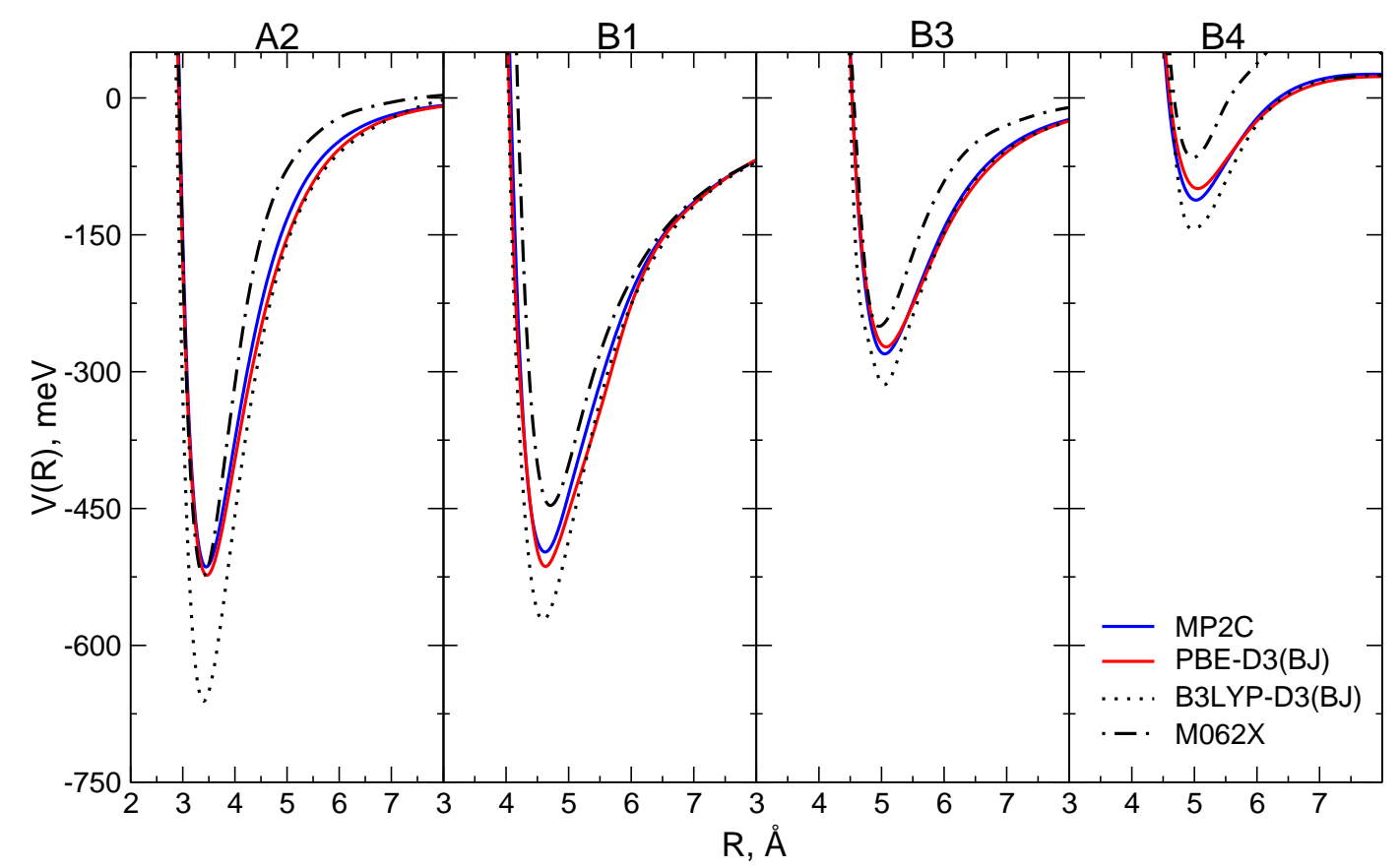

FIG. 4: Comparison of the benchmark MP2C interaction energies with those obtained at the DFT level by using three different functionals, namely PBE, B3LYP and M062X, for the A2, B1, B3 and B4 (see Fig.1) configurations of the CP-pyrene complex. PBE and B3LYP energies have been corrected by adding the D3(BJ) dispersion coefficients of Grimme[49]. 


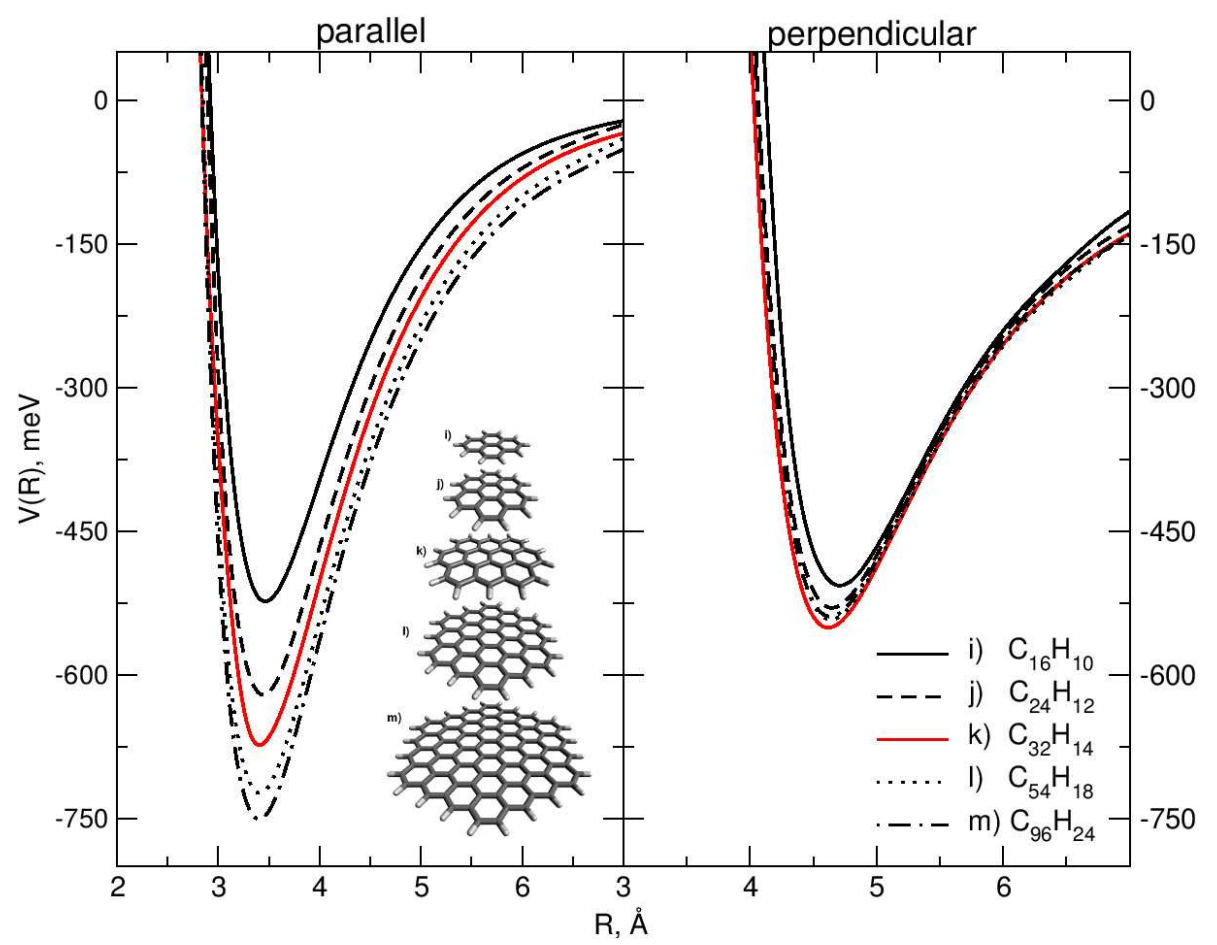

FIG. 5: Energy profiles as a function of the intermolecular distance $R$ obtained at the PBE-D3(BJ) level for the interaction between $\mathrm{CP}$ and different graphene prototypes of increasing size, namely pyrene $\left(\mathrm{C}_{16} \mathrm{H}_{10}\right)$, coronene $\left(\mathrm{C}_{24} \mathrm{H}_{12}\right)$, ovalene $\left(\mathrm{C}_{32} \mathrm{H}_{14}\right)$, circumcoronene $\left(\mathrm{C}_{54} \mathrm{H}_{18}\right)$ and circumcircumcoronene $\left(\mathrm{C}_{96} \mathrm{H}_{24}\right)$. A parallel (left panel) and perpendicular (right panel) configuration, corresponding to the most favourable related approach (see Fig. S2), has been chosen for each graphene prototype. 
a)

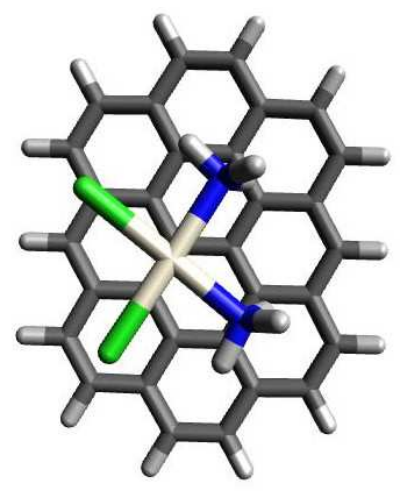

b)
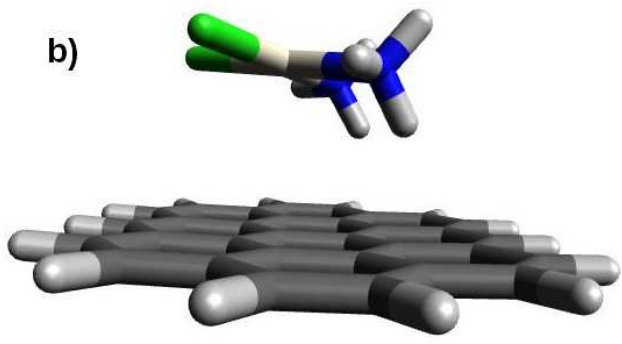

FIG. 6: Top (a) and side (b) view of the most stable configuration of the CP-ovalene complex obtained through a full geometry optimization at the PBE-D3(BJ) level. 